\title{
WEIGHTED VISIBILITY CLASSIFICATION AND SEATING PLAN FOR THE AITKEN UNIVERSITY CENTRE
}

\author{
Tyler Allison, David Fraser, Emmanuel Stefanakis \\ Department of Geodesy and Geomatics Engineering, University of New Brunswick, Canada - (tyler.allison, fraser, \\ estef)@unb.ca
}

KEY WORDS: Facilities, Vision, Visualization, Management, Classification

\begin{abstract}
:
This paper describes the requirements for a weighted visibility classification of seats in the University of New Brunswick's (UNB) Aitken University Centre (AUC). Price levels for seats are typically set for sections of seats based on promoter preferences. In a visibility classification, the digital elevation model (DEM) is created for the AUC and includes possible view obstructions. The view obstructions taken into account for this design were the penalty boxes, player bencher, and the rink boards. There were no other major obstructions in the AUC. The visibility calculations compute the number of visible pixels of the rink surface for each seat. It is expected that seats with a higher number of visible pixels will also have better visibility. The number of viewable pixels is weighted by distance to the center of ice surface to account for the preference of seats that are closer to the rink surface. This paper outlines the collection of data, weighted visibility classification method, and the development of information products. There are two main objectives of this weighted visibility classification and seating plan: (a) to demonstrate that a weighted visibility classification is a viable method to classify seats, and that this methodology could be used to set price levels for a venue and (b) create online web applications to suit the functionality for users and venue administrators. The user web application allows the user to pan, zoom and perform limited searches in the interactive map.
\end{abstract}

\section{Introduction}

The Aitken University Centre (AUC) is a multi-purpose sports facility located on the UNB campus. The arena is used primary for hockey, basketball, concerts and also for high school graduations. The overall purpose of this technical report is to develop a multi-purpose GIS for the AUC. There are currently no interactive seating plans of the Aitken center and this type of application would be very useful for people attending functions at the AUC. The current seating plan of the AUC is not useful for users picking the section, row and seat number for a particular event. A more interactive map would allow people to have a better of idea where there seat is located with respect to the arena.

When attending an event at large venue, there are inherently better and worse seats in the venue. Some seats are closer to the activity while others are at a significant distance. There are also some seats which may have obstructed views while others will have relatively unobstructed views. Typically venues will develop price levels based on proximity and the general trend of personal preference. For this project, a weighted visibility classification of the AUC will be completed. This type of classification would permit price levels to be created based on proximity and visibility.

This technical project contains four primary objectives that will be completed. The first object is to use the acquired data to index the seats. This first step involves assigning certain attributes to individual seats that are required for future objectives. The second objective is to complete a weighted visibility classification of the AUC.

The third objective is link a picture of the view of a seat to the seat entity. There is a growing trend in online ticketing sales that when users are purchases tickets online, being able to forecast what their view will be can influence the decision to purchase a ticket. The final objective is to create web applications that could be used to introduce an online ticketing system for the AUC. The web applications created will provide the foundation for such a system, not an implementation ready system.

The software used to complete all of the objectives for this technical project is esri ArcMap 10.1. This software, freely available to UNB students, has the power to complete all of the required objective.

\section{Data}

A cad drawing of the AUC was provided by James Laslie from facilities management at UNB for this technical report. The cad drawing contained seating patterns for all types of events held at the AUC. The seating pattern of interest and the only one used for this project was the revised layout for hockey events. The drawing consisted of annotations and polyline data. The annotations were all text data describing the row, seat number, and section. Each text had an associated geographical anchor point nearby. Figure 1 shows a zoomed in portion of how a section appeared in ArcGIS. The polylines in the drawing were used to separate seats as well as show other rink information. 


\section{SECTION 17}

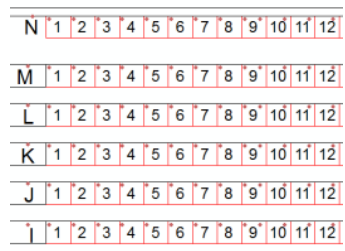

Figure 1: Data from facilities management

\section{Design}

\subsection{Seat Indexing}

The first objective in this project was to index all of the seats. As given, the data is a set of polyline and text information. This information must be combined into a single entity for each seat for querying and classifying purposes. Each seat entity must have the following attributes:

\section{Section Number \\ 2. Row Identifier \\ 3. Seat Number}

\subsection{Visibility Classification}

The computationally intensive section of the project is the weighted visibility classification of all seats in the AUC. The purpose of these calculations is to determine which seats may have partially obstructed views. The classification is based on the amount of viewable pixels from each seat. The viewshed tool is $3 \mathrm{D}$ analyst tool in ArcGIS which can be used to determine the number of viewable pixels from a given observation point. Once the visibility is computed for each seat, seats can classified and symbology maps can be created indicating seats with the best visibility. Seats can also be weighted by distance to provide more realistic classifying.

The purpose of the visibility classification is to determine which seats have an obstructed view. Examples of typical obstruction in hockey venues include player benchers, penalty boxes, scoreboards, and pillars. In the AUC, the only obstructions accounted for are the player benches, the penalty box and the hockey boards. The cad drawing provided the $2 \mathrm{D}$ information and height information was obtained from taking measurements of the boards and using the height of an average hockey player to determine the height of the penalty box and player bench obstructions.

In order to complete a visibility classification, a Digital Elevation Model (DEM) must be created for the AUC. This DEM must include elevations for each seat as well as the obstructions. The cad drawing provided contained only $2 \mathrm{D}$ information therefore the height dimension must be calculated and assigned to entities in the GIS. It was assumed that seats in the same row would have the same elevation consequently it was required to determine the elevation for each row and the obstructions only. Quality Assurance and Quality Control would have to be done on measured data to prove this assumption.
To calculate the number of viewable pixels from each seat, model builder was used to facilitate this computationally intensive and iterative process. The process can be divided into 5 steps. All of these steps lead to the end goal of creating a master table which can be used to link the number viewable pixels to the seat.

Step 1: Viewshed Calculation

Step 2: Clip Rink Surface

Step 3: Index Raster with Object ID

Step 4: Preparation for Append

Step 5: Append

The first tool used in this model is the iterator feature selection tool. This tool allows the model to run for each entity in the seats layer, and outputs a value which is the object ID of the entity currently being iterated. The second tool used is the Viewshed tool. This tool determine the raster surface locations visible to a set of observer features. In this situation, the set of observers is a single seat. This tool uses the imputed raster and determines if each pixel within the raster can be seen from the observer location. Figure 2 shows how this tool was designed in the model builder.

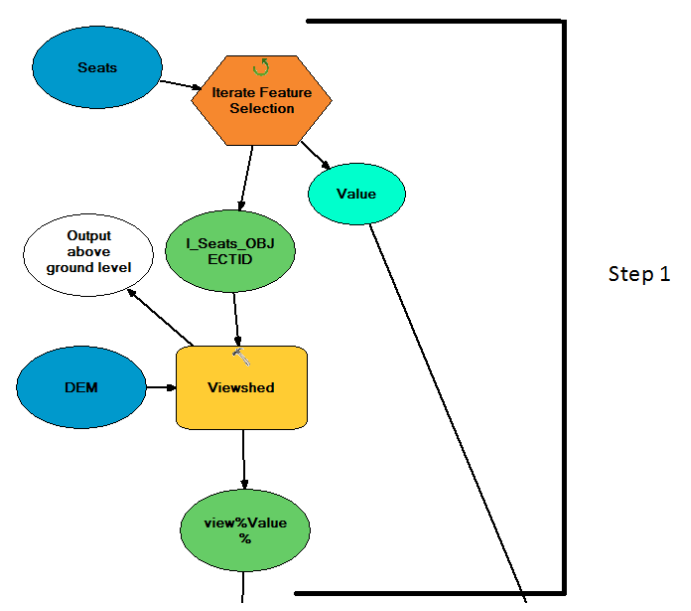

Figure 2: Step 1: Viewshed calculation

The second step in the model is to clip the viewshed raster to the rink surface. As previously discussed, the output raster of the viewshed calculation is the same size of the DEM raster (i.e. includes the seating area). Since the area of interest is the rink surface only, the original raster should be clipped to include on pixels within the rink surface. Figure 3 shows how this step was designed in model builder. 


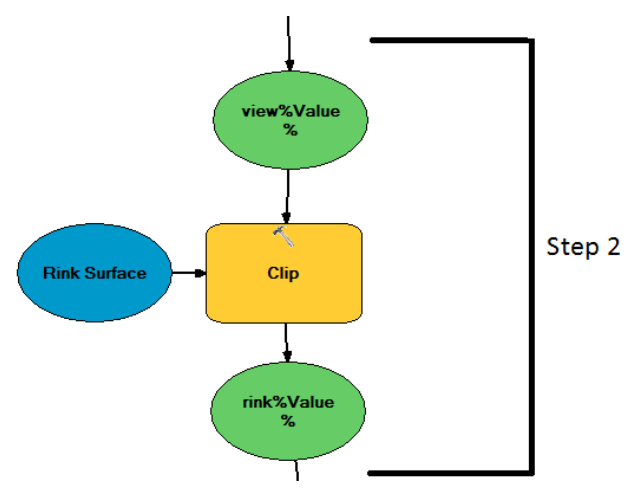

Figure 3: Step 2: clip rink surface

The following three steps are data management steps in order for the final result to contain the appropriate information. Prior to step three, a visibility raster is created and clipped but there is not reference within the raster to a specific seat. In Step 1, the iterative feature selection tool outputs a value which is object. Step 3 adds a field to the raster and assigns the object ID to the table. This step was created using model builder, shown in figure 4. The purpose of this is to have the object ID and the amount of viewable pixels within the same table.

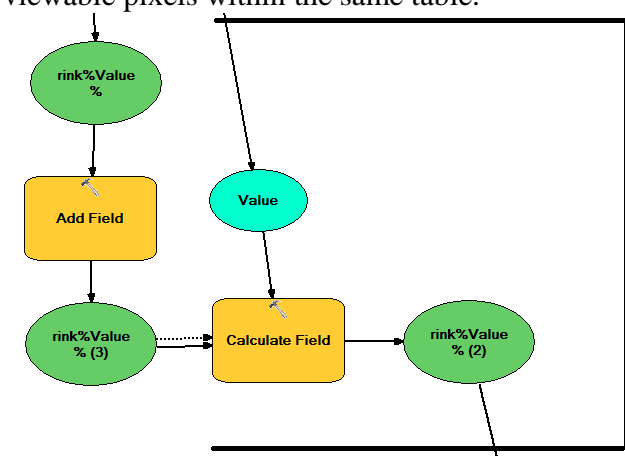

Step 3

Figure 4: Step 3: index raster with object ID

Step 4 is the preparation for appending several tables together, shown in figure 5. At this point in the model, the raster table contains all of the important information and the raster itself is not useful. The table to table tool is used to separate the table from the raster to use this information. The table consists of two rows, the first row showing the number of visible pixels and the second showing the number of pixels which are not visible. This is redundant information because for each iteration the summation of visible and not visible is constant. To reduce confusion and eliminate redundancy, the first row of each table is deleted.

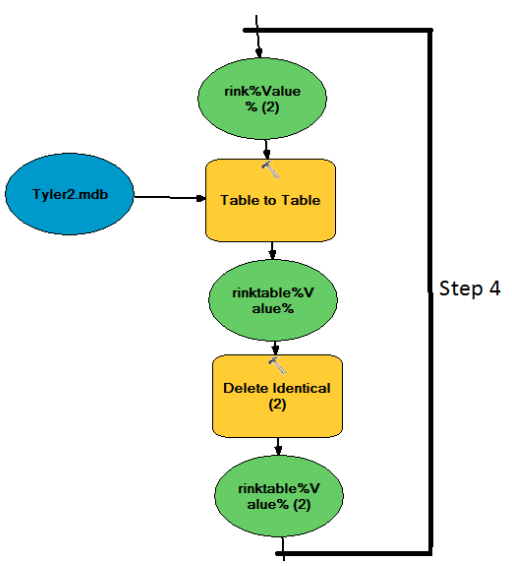

Figure 5: Step 4: preparation for seat append

The final step in the process is to append the table at the end of each iteration. Figure 6 shows the model created to complete this step. This creates a master table which can be used once all the iterations are completed. From this design, the master table contain one row for each seat. Within a row, there is number indicating the number of viewable pixels as well the object ID for the seat.

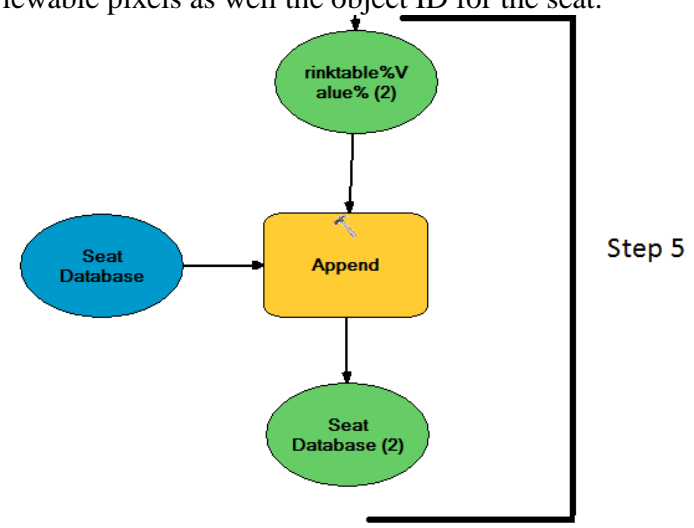

Figure 6: Step 5: append

\subsection{Seat Preview}

It was determined from the literature review that an important functionality for venue maps is to provide a picture suggesting the view from a particular seat. In the design to accommodate this functionality, the picture should be stored online and the URL for the picture should be shown as an attribute for each seat. There are three steps in this design; creating a picture database, assign each seat an attribute to link the seat to the database, and join the picture database to the seat layer.

\subsection{Web Application}

ESRI has several ready to use web application templates that can be used to create maps to publish directly to the Esri cloud. These templates are user friendly but with limited programming skills, functionality is very restricted. In creating web application templates for an online ticketing system, the user and administration require different functionality. Using a different template for each type of user will allow each to be satisfied with the functionality. The templates used are the edit and parcel viewer template. 
The edit web application template provides adequate functionality for the administration of a venue. The key functionality of this template is that it enables editing. It is expected that administration would need to constantly update availability in the venue.

The parcel viewer template is ideal for users and provides good functionality as an interactive map. The parcel viewer is configurable template that enables search and query of attributes in feature layers. The app is responsively designed to support use in browsers, phones, and tablet devices. Being able to search and query is key for users in the AUC interactive map. In the design of the user web template, searches based on availability and visibility are permitted.

\section{Implementation}

\subsection{Seat Indexing}

The first step in the indexing was to convert the data from polylines to features. This was done using the Feature to Polygon tool in ArcGIS. This tool used the enclosed lines in the cad drawing to create polygons.

This tool created a polygon for each seat, but also created hundreds of other polygons as a result of other enclosed lines. The first step to reduce the dataset to include seat polygons only, the geometry was calculated for each seat as an attribute. Using Select by Attribute, only geometry which matched the seat polygons were selected and exported as a new layer. The seat indexing was accomplished using several selection methodologies. A different method was required for the seat number, row index, and section number.

As figure 5.1 shows, each seat number is indicated by an annotation with an anchor point nearby. In the process of creating the polygons for each seat, the anchor point was within the polygon. Using the join data based on spatial location tool, the annotation was added to the attribute table for each seat.

The indexing for the rows was completed by selecting all the seats from a single row, and using the Field Calculator to assign a value to all selected values. To facilitate the process of selecting all the seat from a single, a polyline was drawn around the rink intersecting all the seats in the given row. Using the Select by Location tool, and using intersection as the search criteria, all seats in the row were selected. This process was completed for each row individually.

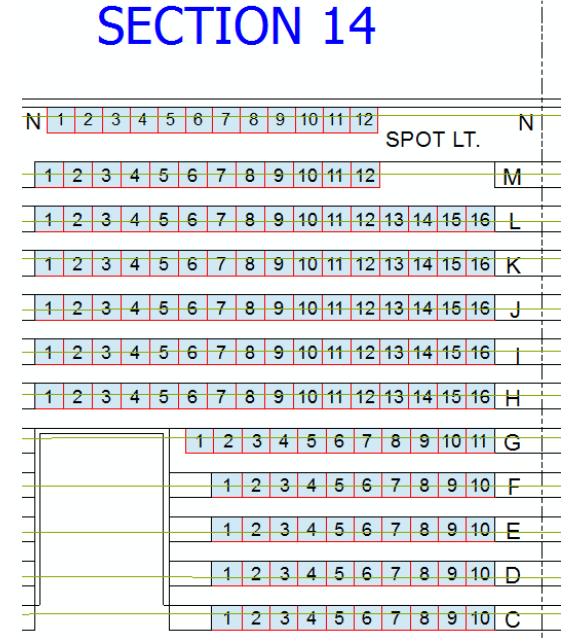

Figure 6: Index rows

The process for index the seats by section was a similar process of selecting all the seats in a section, and using the Field Calculator to assign a number to the selected entities. The process of selected all seats within a section was done using the Select Features tool and drawing a box around a section. This process was completed for each section.

\subsection{Visibility Classification}

\subsubsection{Data Collection}

The first step in the visibility classification implementation was to acquire height information for each row and obstruction. A total station was used the measure heights of each row. For quality assurance and quality control, the heights of each row were calculated on both sides of the venues and the results were compared. The furthest deviation of heights of the same row was 8.5 $\mathrm{mm}$, which confirmed the assumption that rows in the arena are of constant height. Since the height for visibility purposes is of interest and not simply the row height, an additive constant was added to each measured height. The additive constant was based on average distance from the ground in the row to a person's eyes.

To determine the heights of the player bench and penalty box obstructions, the average height of a UNB men's hockey player was used. This value was used as the height of the entire obstruction because a player could be standing anywhere within the bench area. The height used for these obstructions was $2.15 \mathrm{~m}$. The height measurement for the boards obstruction was measured directly, and found to be $0.95 \mathrm{~m}$.

\subsubsection{DEM Creation}

The data used from the DEM creation was seat data and the obstructions. The seat data is considered mass points, which are elevation points that are imported as nodes. The obstructions are defined as hardreplace data, which is used for a polygon datasets that defines areas of constant height. The Create TIN tool was used to create a DEM, shown in figure 7. 


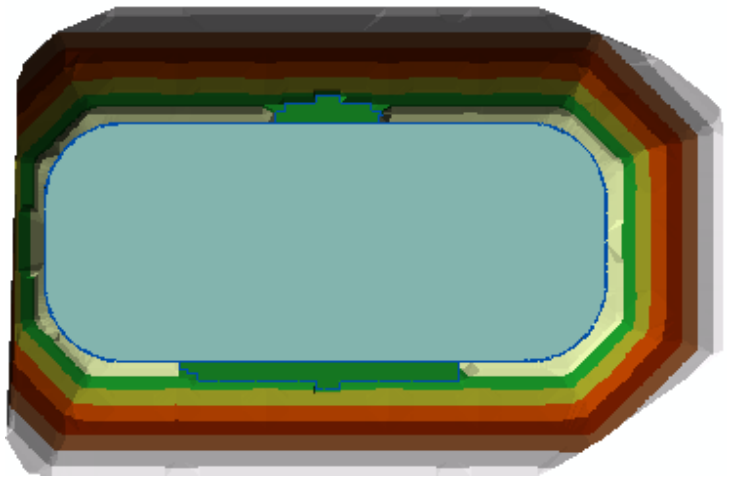

Figure 7: TIN DEM

The TIN was converted to a Raster for the visibility model using the TIN to Raster tool, shown in figure 8.

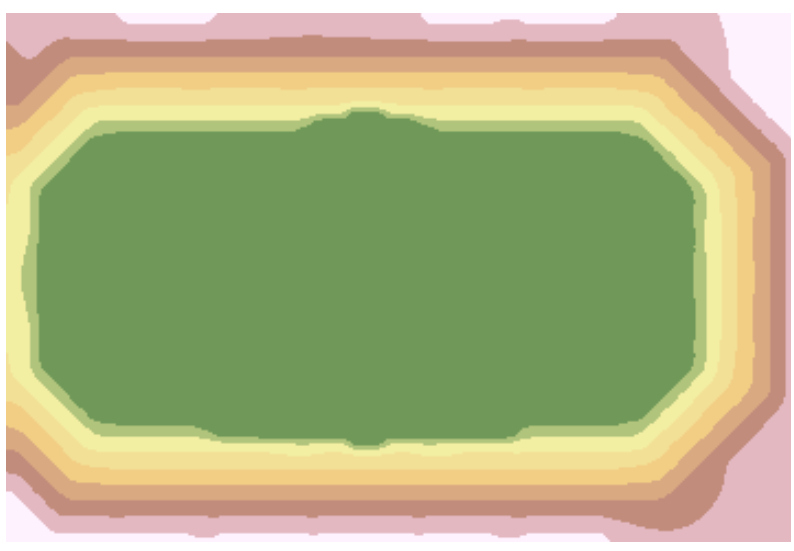

Figure 8: Raster DEM

\subsubsection{Running the Model}

There visibility model must be run for 3152 seats. The model was computationally expensive and whereas the first few iterations would take approximately 5-10 seconds each, after approximately 300 iterations a single iteration would take up to 4 minutes. This problem was likely due to a bug in the system where the computer reached its RAM capacity and could no longer be time efficient. In order to complete the iterations all seats in a timely manner, the following steps were completed until all seats were run through the model.

1. Begin of iteration

2. Let program iterate for 24 hours

3. Stop Iteration and removed completed seats from the dataset

4. Proceed to Step 1

The total running time for the model to complete all 3152 iterations was approximately 100 hours.

\subsubsection{Analysis}

The first step in the analysis was to validate the accuracy of the model. Step 1 and 2 are the creation of visibility raster and steps 3 to 5 are data management operations. It is paramount to ensure that the visibility raster created is accurate to the situation. Figure 9 shows the clipped visibility classification of a seat located in the south west corner of the AUC. In this figure, the blues pixels are visible to the observer and grey pixels are not. It is expected that seats in this area would not have an obstructed view to the north side of the rink but would likely have difficulty seeing the south corner as well as other portions on the south side of the rink. Several visibility raster's were checked to ensure the model was running as expected. There were no major detected blunders in the model.

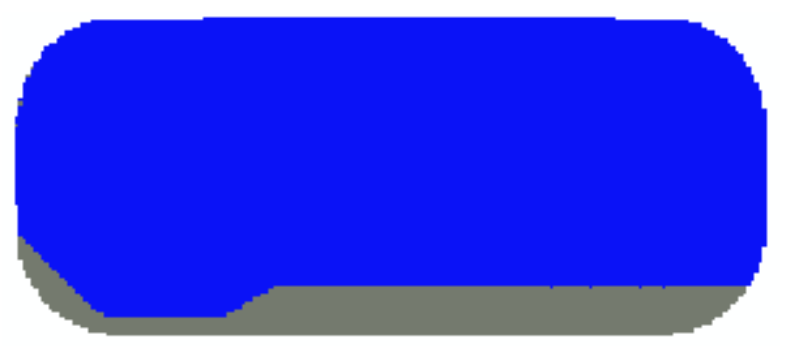

Figure 9: Visibility raster clipped

Once the model was completed and the quality of the raster was checked, the master table was linked to seat dataset using the object ID as the join criteria. Once the number of visible pixels was linked to the individual seat, visibility classification maps could be created. Figure 10 shows the visibility classification for the entire AUC and the legend for the map. This figure shows the trend that seats located at the ends of the arena are not as obstructed as seats in the middle portion of the rink which are obstructed by the penalty box and player benches.

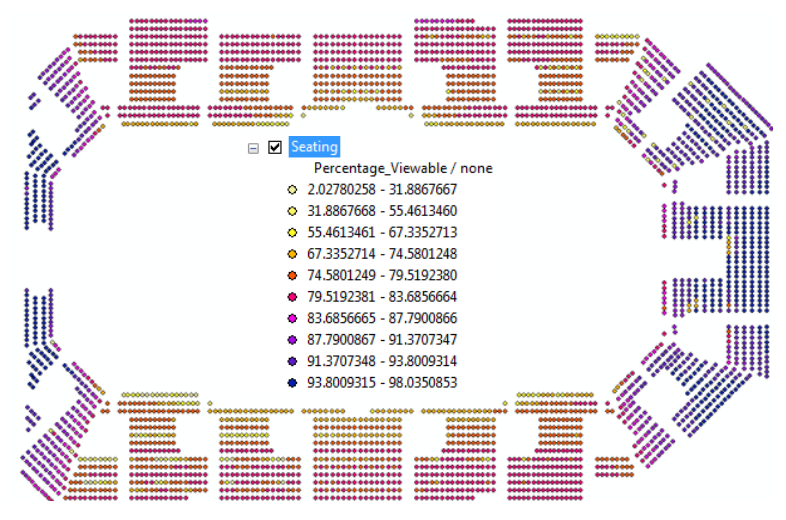

Figure 10: Visibility classification

There are two conditions which are important to a person going to a hockey game; visibility and proximity. The above analysis shows which seats have the best visibility but are not weighted by distance. To give an accurate result of the seat classification, the visibility must be weighted by distance. To accomplish this task, the below equation is used. Although the numeric value of this equation is arbitrary, it is the relative relationship between seats that is important. This equation was used because the weighting of distance to visibility was found to best describe the relationship.

Seat Classification $\propto \frac{\# \text { of Viewable Pixels }}{\text { Distance to Centre Ice } \text { I }^{1 / 4}}$ 


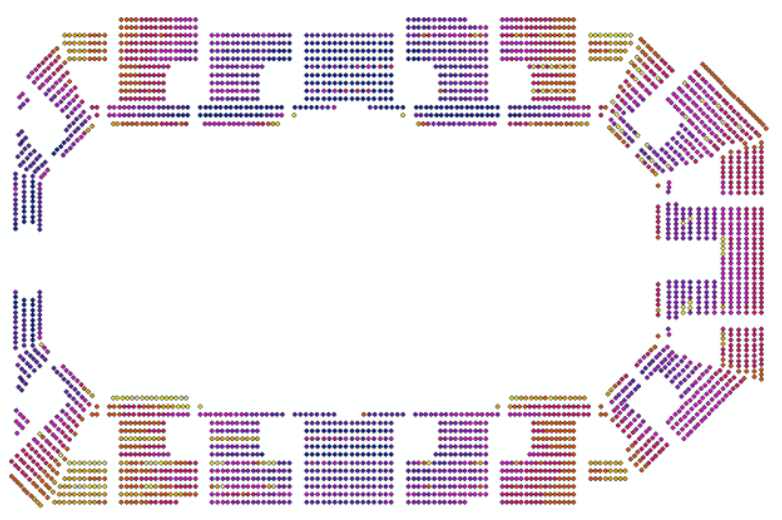

Figure 11: Weighted visibility classification

\subsection{Seat Preview}

There are three steps in the seat preview implementation; creating a picture database, assign each seat an attribute to link the seat to the database, and join the picture database to the seat layer.

The image hosting site used for this project is Imgur, chosen because it is free and user friendly. In the design of the picture database, a table was created with two attribute fields. The first is picture description and the second is the URL for the picture. The picture description indicates where this picture is taken. Figure 12 shows the database, where the first row description shows the picture was taken in section 1 , in the front. Since there are only two picture taken per section, only the distinction of front and back is required within a section.

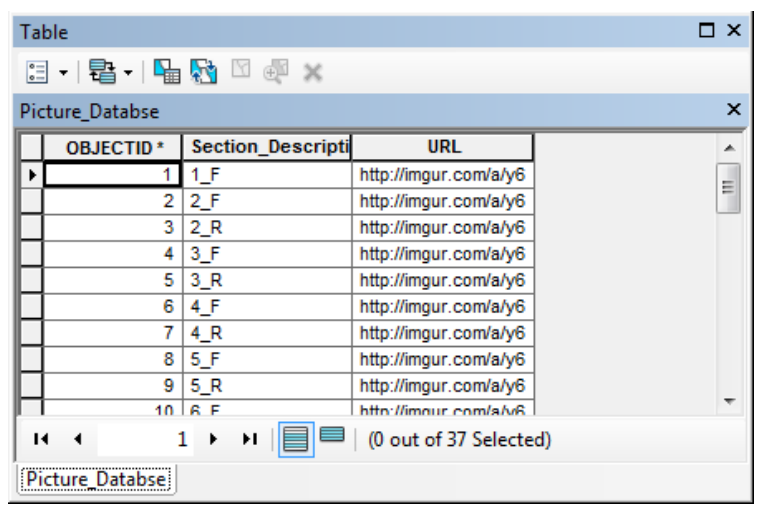

Figure 12: Picture database design

The second step in the design to assign each seat a description which can be used to link the picture database to the seat. Figure 13 shows the model used to complete this task. In the Calculate Field tool, a seat description is assigned to the selected seats with corresponds to the seat descriptions in the picture database. This task was iterated for each picture but required a small number of manual changes between iterations. In larger venues with significantly more sections, it may be more time efficient to automate the process to remove any required manual actions.

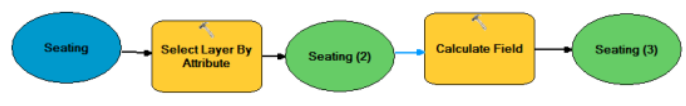

Figure 13: Assigning Picture Descriptor to Seat

The previous steps provide a link between the picture database and the seats dataset. Both sets of data contain a section identifier. Using the join attributes from table tool, with the seat description field as the join criteria, the tables are joined. Once the tables are joined, each seat has attribute which contains a URL with a view from that area. ArcGIS allows the option to recognize a field as a URL. With this option set, simply clicking on a seat in ArcGIS desktop will open the URL, showing the preview of the seat.

\subsection{Web Application}

The purpose of a web application is to provide user friendly functionality. The first step in this process was to only include certain attributes in the seats database. The following table shows the attribute table for the seats layer which would be shared on ArcGIS online. The seat quality attribute was modified to be easy for users to understand. Where an arbitrary number for the visibility classification would be meaningless, indicating the percentile which a seat is given easier for users to understand. In Figure 14, the seats quality indicates the seats are in the top 50\%. The symbology for the service to be shared online is based on availability because this is the most important factor for both users and administration. Seats which are available are a black colour and seats which are unavailable are shown in light grey. The dataset was georeferenced to allow for a variety of basemaps to be shown with the data. A blank basemap was used in for both web applications made to reduce map clutter.

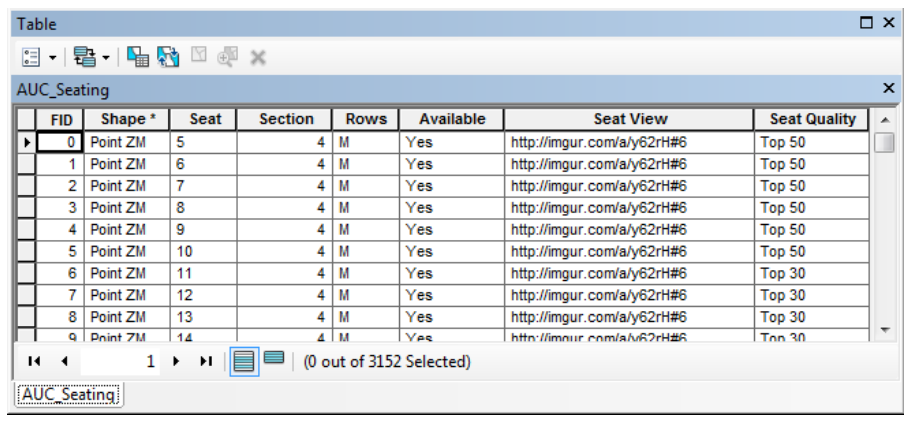

Figure 14: Reduced seat attribute table

In ArcGIS desktop, the program has no difficulty drawing all of the entities very quickly. When working from an online server the seats dataset is too large and at a zoom level showing the entire rink, some seats will fail to draw. For this reason, polygons are drawn around sections and zoom levels are introduced as a way get by this problem. Figure 5.10 shows the map drawn at two scales; 1:125 and $1: 100$. 


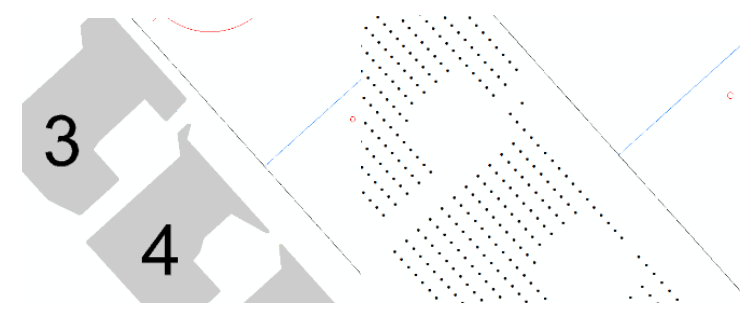

Figure 15: Zoom levels

It is important to note that both web applications were created using the same dataset. This allows any changes in the edit template to be automatically updated in the user application. This is particularly useful when selling tickets for a venue event so that the data is continually updated for the user.

\subsubsection{Edit Template}

For administration purposes, the edit web application template was the best option. The advantage of this template was that it allowed easy editing of seat availability. Since the server is hosted online, multiple computers could be used at the same time. Figure 5.11 shows the template. The edit template is available at http://unbfred.maps.arcgis.com/apps/Edit/index.html?app id $=c 81 b b 18 \mathrm{e} 01 \mathrm{c} 34 \mathrm{fbeac} 2154 \mathrm{e} 0 \mathrm{e} 0 \mathrm{~d} 27 \mathrm{~b} 08$.

\subsubsection{Parcel Viewer Template}

For user purposes, the parcel viewer template provided the most amount of functionality. User can view seat attributes as well as search based on the configured parameters. Figure 5.12 shows the result of clicking a seat. Clicking more info on URL attribute with open a new webpage showing a picture from the area where the seat is located. The search criteria is a configurable parameter for this template. The search criteria available in this template is for availability and seat quality. If a user wishes to see where top $2 \%$ (in term of quality) of the seats are located, entering "Top 2" in the search bar will return all seats that match this criteria. In the typical situation where there may be limited availability, user can search "Yes" into the search bar to return seats that are still available. The parcel viewer template is available at http://unbfred.maps.arcgis.com/apps/Solutions/s2.html?a ppid=e179382fa35a4fbda87ff68a187f4011.

\section{Conclusion}

This research project was set out to create web applications for the Aitken University Centre. The current seating plan is out of date and with the GIS tools available today, it should be updated. This project reached its major goals of providing online web applications. The second component of this project involved a weighted visibility classification. The results showed that a classification could be done quantitatively. This type of classification could be used to assign accurate price levels in a venue. This type of classification would be most useful in a venue with several obstructions.

The process of completing the objectives for this technical report was a tremendous learning experience but not without several obstacles. Many obstacles were a result of a lack of experience with the ArcGIS software. In many cases, it was a trial and error process to determine the most time efficient and accurate method to complete a task. ArcGIS has hundreds of available tools and with several potential methods to solve a problem, choosing the correct tools is a task within itself.

There are several expansions that could be made in the future. The anticipated expansions are related to the web applications as more functionality would be an asset, particularly in the user application. The templates used for this project were ready to use templates with very limited configurable parameters. With the proper skills in computer programming, functionalities such as querying and more in depth searches could be completed. It would be ideal for a user to search for section, row, and seat to locate a specific seat. These capabilities are not possible with the current web application because only a single search can be completed.

\section{ACKBOWLEDGEMENTS}

I would like to thank Dr. Emmanuel Stefanakis and David Fraser for their continuous support and providing with the opportunity and resources to complete this technical report. Without their support, this project would not have been possible. I would like to expressly thank David Fraser, whom was instrumental in overcoming any ESRI ArcGIS related problems.

\section{REFERENCES}

ESRI [2013]. Using Hyperlinks, ArcGIS Help 10.1 Resource Centre. [On-line] 20 November

2013.http://resources.arcgis.com/en/help/main/10.1/index .html\#//00s5000000 04000000

Leslie, J [2013]. Personal Communications. Drafting Coordinator, Facilities Management, UNB Fredericton

Fraser, D [2013]. Personal Communications. Technical and Scientific Staff, Geodesy and Geomatics Engineering, UNB Fredericton

Lu L., Paulovicks B, Perrone M, Sheinin V. [2011] High Performance Computing of Line of Sight Viewshed. T.J. Watson Research Centre, New York.

Payne, Andrew [2008]. Seat Selection Method for Event Tickets, U.S. Patent Application Publication No. $12 / 118,391$

Riggs P.D. and Dean D.J. [2007] An Investigation into the Causes of Errors and Inconsistencies in Predicted Viewsheds. Department of Geospatial Sciences, Colorado State University

Sander, H. A. and S.M. Manson [2007]. Heights and locations of artificial structures in viewshed calculation: How close is close enough? Minnesota, USA

Stekanakis, E., [2013]. Personal Communication. Full Time Faculty, Geodesy and Geomatics Engineering, UNB

Sunshine, Steven A., Rod Goodman, Michael Arya, Larry Chu, and Michael Ripberger [2013]. Sports and 
The International Archives of the Photogrammetry, Remote Sensing and Spatial Information Sciences, Volume XL-2, 2014 ISPRS Technical Commission II Symposium, 6 - 8 October 2014, Toronto, Canada

Concert Event Ticket Pricing and Visualization System, U.S. Patent Application Publication No. 13/742,296 University of New Brunswick [2013]. Aitken University Centre Seating Plans. [On-line] 20 November 2013. http://www.unb.ca/fredericton/kinesiology/facilities/auc /seatingplan.html 